\title{
The Prevalence of Asymptomatic Cervical and Lumbar Facet Arthropathy: A Computed Tomography Study
}

\author{
Jung Hwan Kim ${ }^{1}$, Alok Sharan ${ }^{2}$, Woojin $\mathrm{Cho}^{3}$, Mohammed Emam ${ }^{4}$, Michael Hagen ${ }^{5}$, Soo Yeon Kim ${ }^{5}$ \\ ${ }^{1}$ Department of Anesthesiology, Perioperative, and Pain Medicine, Icahn School of Medicine at Mt. Sinai St. Luke's and Mt. Sinai West Hospitals, \\ New York, NY, USA \\ ${ }^{2}$ Department of Orthopedic Surgery, Westmed Medical Group, Yonkers, NY, USA \\ ${ }^{3}$ Department of Orthopedic Surgery, Montefiore Medical Center, Albert Einstein College of Medicine, Bronx, NY, USA \\ ${ }^{4}$ Department of Physical Medicine and Rehabilitation, SUNY Downstate Medical Center, Brooklyn, NY, USA \\ ${ }^{5}$ Department of Rehabilitation Medicine, Montefiore Medical Center, Albert Einstein College of Medicine, Bronx, NY, USA
}

\section{Study Design: Case control study.}

Purpose: To determine the prevalence and degree of asymptomatic cervical and lumbar facet joint arthritis. We retrospectively reviewed 500 computed tomography (CT) scans of cervical facet joints obtained from 50 subjects. Moreover, 500 lumbar facet joints obtained from an additional 50 subjects were reviewed.

Overview of Literature: Numerous reports in the literature indicate that joint arthritis is a major source of axial neck and low back pain. However, the diagnostic value of this condition, based on degenerative changes seen on radiological studies, remains controversial because significant imaging findings may not correlate with corresponding symptoms. The CT scan is a sensitive method for facet joint evaluation and may reveal degenerative abnormalities. Previous studies have described the prevalence of facet arthropathy in symptomatic patients, according to radiological findings; however, no study to date has assessed its prevalence in asymptomatic patients.

Methods: We retrospectively reviewed the neck and abdominal CT scans of patients had been examined for non-spinal pathologies (i.e., thyroid disease, rule out cancer, ascites). Electronic medical records were reviewed to exclude patients with histories of either neck or back pain. Arthritis severity was graded using a previously published four-point CT scale.

Results: The prevalence of asymptomatic cervical facet arthritis (grade 1-3) was 33\% (grade 1, 19\%; grade 2, 11\%; and grade 3 , $3 \%$ ). Among asymptomatic patients, $37 \%$ had scalable lumbar facet join arthritis (grade 1,24\%; grade 2, $9 \%$; and grade 3, 4\%). There was a statistically significant difference (chi-square test, $p<0.0001$ ) in the number of older individuals with arthritic degeneration at the cervical and lumbar levels compared with that of younger individuals. The C6-C7 and L5-S1 levels were the most likely to show arthritic changes.

Conclusions: Arthritic changes to the cervical and lumbar facet joints are prevalent among patients, and in some cases are asymptomatic. These findings were more common in older patients and at lower spinal levels.

Keywords: Facet joint; Zygapophyseal joint; Cervical spine; Lumbar spine; Diagnostic imaging; Prevalence study 


\section{Introduction}

Chronic neck (CNP) and lower back pain (CLBP) are among the most common musculoskeletal complaints in the United States and affect approximately $66 \%$ [1] and $84 \%$ [2] of the population during their lifetime, respectively. Neck and back pain impose a large burden on patients and society, with an estimated cost of approximately $\$ 87.6$ billion in 2013-a cost surpassed only by diabetes and ischemic heart disease [1].

Multiple factors can cause CNP and CLBP, including disorders of the intervertebral discs (IVDs), facet joints, ligaments, muscles, and spinal nerve roots. Because of this, diagnosis can be difficult. Axial neck and back pain are believed to be caused by facet arthropathy, rather than nerve root irritation, as suggested by studies that have induced neck and back pain via chemical and mechanical stimulation of the facet joints [2-4].

There is an increasing prevalence of CNP and CLBP with age, which can be partially attributed to IVD degeneration. The degenerative cascade, initiated at the level of the IVD, ultimately increases facet joint loading and contributes to subsequent development of facet arthritis. In cases wherein computed tomography (CT) imaging has shown degenerative changes of the facet joints in patients with a history of CNP or CLBP, the ensuing treatment recommendations often include facet joint injections [2,5,6]; however, Marks et al. [2] have reported that only one-tenth of those who received injections actually experienced CLBP symptom relief. This study suggested that observation of facet joint degeneration using imaging studies alone may contribute to incorrect diagnosis of facet syndromes, and lead to unnecessary and ineffective treatments in the form of facet joint blocks. Furthermore, diagnostic injections are not reliable. Manchikanti et al. [7] have shown a low response rate to intraarticular steroid injections, and a false positive rate of $25 \%$ associated with diagnosis of facet syndrome.

The introduction of motion-preserving spinal implants (total disc replacements) has focused additional attention facet joint arthritis. These implants are intended to alleviate painful IVD pathologies that are often accompanied by facet joint arthritis [8], a contraindication for implant placement. A better understanding of the prevalence of facet joint arthritis may help spinal surgeons recognize those who are ineligible for motion-preserving implants.

Studies on nerve blocks, CNP, CLBP, and whiplash- associated disorders have examined the prevalence of facet arthritis in symptomatic patients $[9,10]$. Other studies have determined the prevalence of facet arthritis in cadavers [11]; however, to date there have been no studies that examined the prevalence of facet joint arthritis in 'asymptomatic' patients. Therefore, our study sought to establish the prevalence and degree of arthritis-related facet joint changes in asymptomatic patients. Our study objective was to better understand the prevalence of facet joint arthritis in asymptomatic patients as a means of better quantifying the number of individuals who do not respond to facet injections.

\section{Materials and Methods}

\section{Subjects}

After obtaining institutional review board approval, the institutional picture archiving and communication system (PACS) database (GE Centricity, Milwaukee, WI, USA) was queried to identify patients who underwent imaging studies for non-spinal clinical indications. Two distinct cohorts were generated from patients scanned between 2009-2010. These included (1) patients who underwent
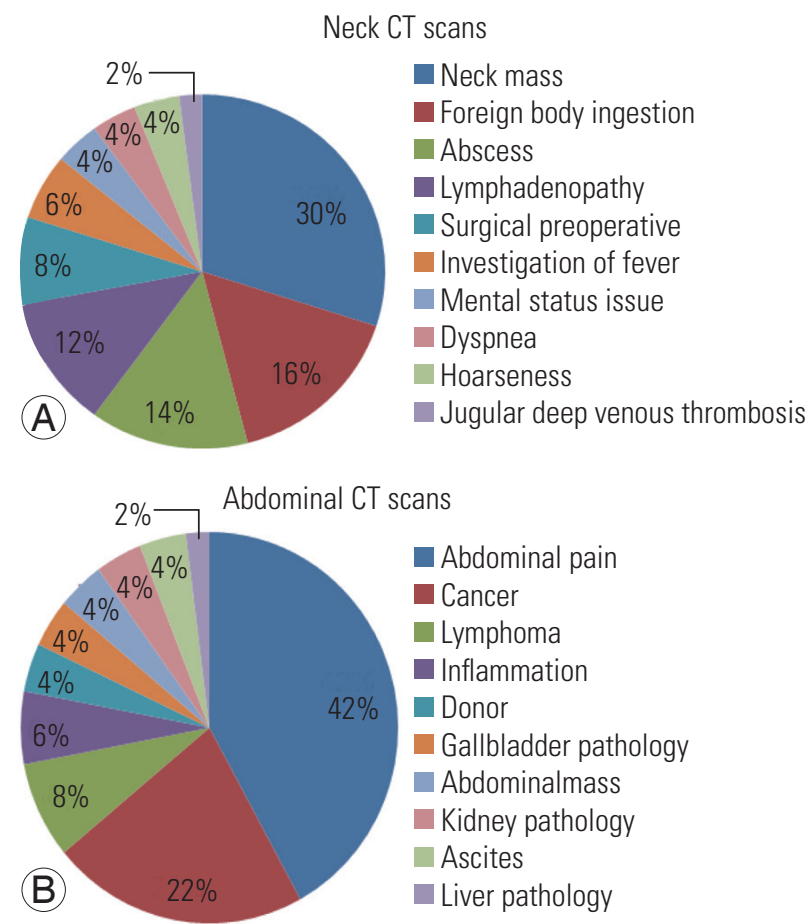

Fig. 1. Non-spinal pathology indications for CT scans for (A) neck pathologies and (B) abdominal and pelvic pathologies. CT, computed tomography. 
neck CT imaging for cervical facet joint examination and (2) patients who underwent abdomen/pelvis CT imaging for lumbar facet joint examination (Fig. 1). Thin-section images $(0.625 \mathrm{~mm})$ were obtained in all cases, using a helical, 64-slice technique. We ultimately identified 4,490 patients with neck CT imaging and 3,328 patients with abdominal and pelvic CT imaging. Inclusion criteria were: 20-69 years in age and adequate visualization and grading of all facet joints. We excluded patients with evidence, or histories, of neck or back pain, patients seen by a spine surgeon for a treatment of neck or back pain, patients with a history spinal fracture, and those with any evidence of prior spine surgery. Studies were selected from their respective cohorts in sequential, chronological order starting from the most recent CT examinations. We established five target age groups (20-29, 30-39, 40-49, 50-59, and 60-69 years) based upon the typical age range in which patients may be considered for treatment with facet joint block or disc arthroplasty, then selected 10 patients within each age group who met all inclusion criteria. Thus, a total of 500 cervical facet joints in 50 patient subjects and 500 lumbar facet joints from another 50 patient subjects were reviewed.

Patients were considered asymptomatic if they had no records of prior cervical or lumbar imaging (X-ray, magnetic resonance imaging, or CT scan) in our PACS database. In addition, we comprehensively reviewed each patient's electronic medical record data prior to inclusion, in order to assure that all patients met the pre-specified inclusion and exclusion criteria. Overall, 19 males and 31 females were included in the cervical facet review, whereas 26 females and 24 males were included in the lumbar spine review.

\section{Image evaluation}

CT Images were independently graded by an orthopedic spine surgeon, board-certified neuroradiologist, and a specially trained medical student using the Centricity PACS Workstation (GE Centricity). Three observers with different training backgrounds reviewed the facet joints to eliminate training bias. The method for grading the severity of facet joint arthritis symptoms was adapted from Weishaupt et al. [9] (Fig. 2) and is summarized in Table 1.

\section{Statistical analysis}

Chi-square tests were used to compare data pertaining to
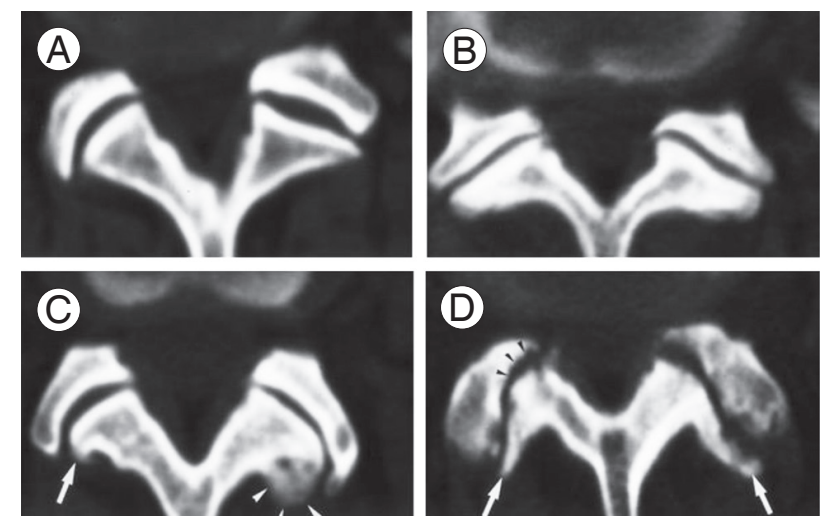

Fig. 2. Criteria for grading arthritis of the facet joints. Adapted from Weishaupt et al. Skeletal Radiol 1999;28:215-9, with permission of Springer Science+Business Media [9]. (A) Grade 0, (B) grade 1, (C) grade 2, (D) grade 3. Disregard arrows in (C) and (D).

Table 1. Criteria for grading severity of arthritis in facet joints

\begin{tabular}{ll} 
Grade & \multicolumn{1}{c}{ Criteria } \\
\hline 0 & No narrowing, sclerosis, or osteophytes \\
1 & Joint space narrowing or irregularity \\
\hline 2 & $\begin{array}{c}\text { Narrowing plus sclerosis and/or hypertrophy with } \\
\text { osteophyte formation }\end{array}$ \\
3 & $\begin{array}{c}\text { Complete narrowing, sclerosis, and/or severe osteophyte } \\
\text { formation }\end{array}$ \\
\hline
\end{tabular}

Adapted from Weishaupt et al. Skeletal Radiol 1999;28:215-9, with permission of Springer Science+Business Media [9].

arthritic changes and non-degenerative facet joints across the different age groups. Two sample $t$-tests were used to test significant age-related differences in arthritis (at each joint level). Grades 1, 2, and 3 were combined to form an arthritic degeneration group. The data reported in this paper represents the average of the results of the three reviewers. We calculated Cohen's kappa coefficient to determine inter-observer reliability.

\section{Results}

\section{Cervical data}

We examined a total of 500 cervical facets, from $50 \mathrm{pa}-$ tients. The weighted kappa coefficient for inter-observer reliability was 0.5 , indicating a moderately strong positive agreement. The prevalence of facet arthritis by grade is summarized in Table 2. Asymptomatic cervical facet joint arthritis was observed in $33.4 \%$ of patients. Over half of those with evidence of arthritic changes (57\%) demon- 
Table 2. Prevalence and severity of arthritic changes in cervical and lumbar facet joints

\begin{tabular}{ccc} 
Grade & Cervical & Lumbar \\
0 & $333(67)$ & $320(63)$ \\
1 & $93(19)$ & $118(24)$ \\
2 & $57(11)$ & $44(9)$ \\
3 & $17(3)$ & $18(4)$ \\
\hline
\end{tabular}

Values are presented as number of arthritic joints (\%). The prevalence of asymptomatic cervical facet arthritis was 33\% and lumbar facet arthritis was $37 \%$.
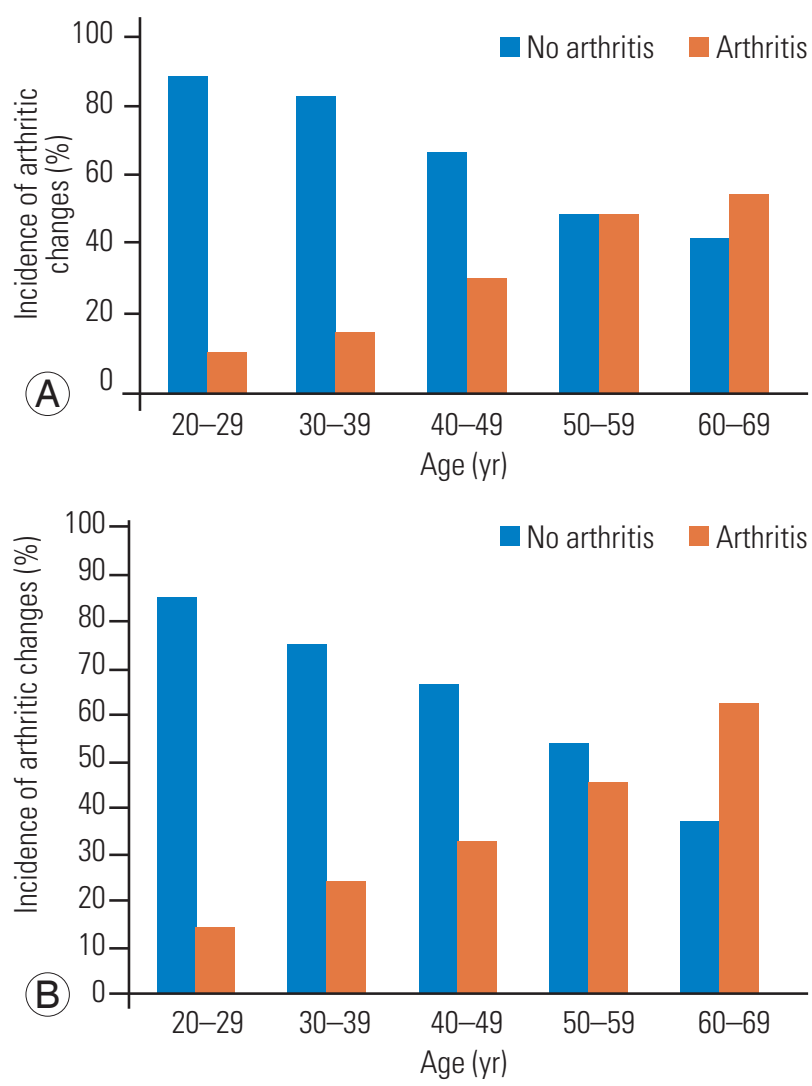

Fig. 3. The incidence of no arthritic changes (grade 0) decreases with age, whereas arthritis (grades 1,2,3) increases among the different age groups. (A) Arthritis in cervical facet joints and (B) arthritis in lumbar facet joints.

strated only mild joint space narrowing or irregularities (grade 1). The prevalence of no arthritic changes (grade 0 ) and arthritic changes (grade 1,2,3) among the different age groups are displayed in Fig. 3A. Approximately half of the facet joints $(46 \%)$ that were analyzed in patients older than age 40 showed signs of arthritic changes. The prevalence of normal, non-degenerated facets decreased with age, indicating that the prevalence of arthritis increases with age. A statistically significant difference (chi-square
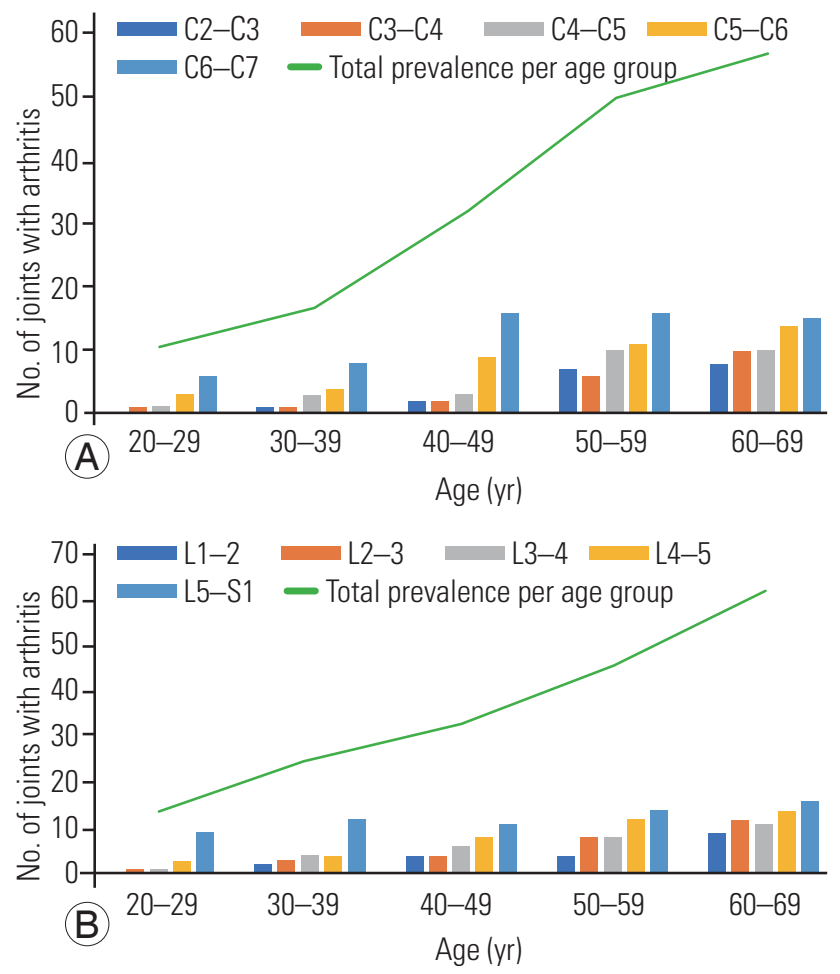

Fig. 4. Each bar represents the number of facet joints at each spinal level, per age group, with signs of degeneration (grades 1, 2, 3). (A) Cervical facets with arthritis and (B) lumbar facets with arthritis.

test, $p<0.0001)$ was observed in the prevalence of arthritic degeneration, at all cervical levels, among the age groups.

At each cervical level, there was a significant increase in arthritic changes in patients older than 45 , compared to younger patients. All values were statistically significant $(p<0.050)$. Within each age group, there was a general trend toward greater arthritic changes at the more caudal spinal levels (Fig. 4B). Facet joint arthritis of the C6-C7 level was found in $78 \%$ of patients older than 40 years, compared to only $29 \%$ at the C2-C3 spinal level within the same age group.

\section{Lumbar data}

Here, we examined a total of 500 cervical facets from 50 patients. The weighted kappa coefficient for interobserver agreement was 0.41 indicating a borderline moderately strong, positive agreement. Asymptomatic lumbar facet joint arthritis was observed in 37\% of patients (Table 2). Two-thirds of the subjects had grade 1 arthritic changes. Across the age groups, there was a trend towards increasing degenerative changes with increasing age (Fig. 3B). Statistically significant differences (chi-square test, 
$p<0.0001)$ were observed in the prevalence of arthritic degeneration, at all lumbar levels, among all age groups. When comparing lumbar arthritis prevalence at each level for the different age groups, there were significantly more degenerative changes $(t$-test, $p<0.05)$ in the facet joints of patients older than age 45 , compared to younger patients. There was a trend of increasing arthritic degeneration at caudal levels like L5-S1, compared to cephalad levels (Fig. 4B). For example, examining the facet joints of patients younger than 50 years, $54 \%$ exhibited arthritic changes at the L5-S1 levels. In contrast, only $12 \%$ of the same group exhibited arthritic changes at the L1-L2 levels.

\section{Discussion}

The prevalence of CNP and CLBP increases with age, producing significant morbidity and disability. Understanding the etiology of pain can help practitioners determine appropriate treatments. Facet joint degeneration is associated with increasing age-related joint loading [9]. In this study, we attempted to document the prevalence of cervical and lumbar facet arthritis in asymptomatic males and females.

Arthritic changes to the facet joints were especially prevalent among patients older than 45 years. In this age group, we found asymptomatic cervical facet arthritis in $33 \%$ of patients and lumbar facet arthritis in 37\%. Arthritic degeneration also appeared more frequently in the facet joints of more caudal levels, with progressively greater arthritic changes in the facet joints from $\mathrm{C} 2-\mathrm{C} 3$ to $\mathrm{C} 6-$ C7. This trend held for each age group that was analyzed in this study and is intuitive as the lower spinal segments bear greater axial loads. Overall, the prevalence of lumbar facet joint arthritis was greater than cervical facet joint arthritis.

In this study, we were able to demonstrate that asymptomatic cervical and lumbar facet arthritis is common, occurring in approximately one-third of our patient population. The high prevalence of this condition in seemingly asymptomatic patients indicates that CT might be a nonspecific diagnostic modality for neck or low back pain, originating from the facet joint. High-resolution singlephoton emission computed tomography (SPECT) may be an even better diagnostic tool, because of its ability to detect inflammation [12]. Evaluation of asymptomatic facet arthritis and clinical symptoms can be predicted by the presence of inflammation and is distinct from narrowed or hypertrophic facet joints, which are likely painless. The use of facet block injections after a positive SPECT analysis should decrease the number of false-positive diagnoses of facet syndrome as a source of CNP and CLBP.

Pain relief from intraarticular steroid injections occur by blocking prolonged nociceptive discharge triggered by facet joint capsular stretch, like what occurs during a whiplash accident [13], rather than from pain due to arthritis. For these cases, CT imaging is less able to discriminate patients who would benefit from steroid injections, since any arthritic changes to the facet joint arthritic would likely be coincidental and noncontributory.

We found that arthritic changes within facet joints are relatively common, especially among patients older than 45 years. Therefore, motion-preserving implants may be more suitable for younger patients, since facet joint arthritis is a contraindication for such implants.

Finally, we found greater arthritic degeneration in the facet joints of caudal spinal levels, with progressively greater arthritic changes in the facet joints from $\mathrm{C} 2-\mathrm{C} 3$ to C6-C7. This trend held for each age group that was analyzed in this study and is intuitive as the lower spinal segments bear greater axial loads. There was an overall greater prevalence of lumbar facet joint arthritis compared to cervical facet joint arthritis.

One limitation of this study was its retrospective design. Patients were not directly queried regarding the presence of pain, which may have been mild or otherwise not documented in their electronic medical record at our institution. However, this was mitigated since many patients at our institution receive coordinated medical care though our institution's broad range of medical and surgical clinics.

Further studies are needed to better distinguish between symptomatic and asymptomatic facet joint arthritis. SPECT can be an alternative means of detecting inflammation as a potential source of pain; however, arthritis alone can cause pain and therefore less invasive methods are needed to evaluate facet syndrome, rather than proceeding immediately to a trial of steroid injection treatments.

\section{Conclusions}

We evaluated the CT scans of 500 cervical facet joints, obtained from 50 patients, and 500 lumbar facet joints from another 50 patients. The prevalence of asymptomatic 
facet arthritis was 33\% in cervical facet joints and 37\% in lumbar facet joints. Our findings suggest that arthritic changes in the cervical and lumbar facet joints are prevalent in asymptomatic patients and are progressively more common with increasing age and at lower spinal levels.

\section{Conflict of Interest}

No potential conflict of interest relevant to this article was reported.

\section{ORCID}

Jung Hwan Kim: https://orcid.org/0000-0001-5558-5016

Soo Yeon Kim: https://orcid.org/0000-0001-9433-4121

\section{Author Contributions}

Writing the article: Jung Hwan Kim; conception and design: Alok Sharan; provision of materials, patients, and resources: Woojin Cho; analysis and interpretation: Mohammed Emam; revision of the article: Michael Hagen; and critical revision of the article and final approval of the article: Soo Yeon Kim

\section{References}

1. Dieleman JL, Baral R, Birger M, et al. US spending on personal health care and public health, 1996-2013. JAMA 2016;316:2627-46.

2. Marks RC, Houston T, Thulbourne T. Facet joint injection and facet nerve block: a randomised comparison in 86 patients with chronic low back pain. Pain 1992;49:325-8.

3. Dwyer A, Aprill C, Bogduk N. Cervical zygapophyseal joint pain patterns: I. a study in normal volunteers. Spine (Phila Pa 1976) 1990;15:453-7.
4. O'Neill S, Graven-Nielsen T, Manniche C, ArendtNielsen L. Ultrasound guided, painful electrical stimulation of lumbar facet joint structures: an experimental model of acute low back pain. Pain 2009;144:76-83.

5. Cohen SP, Hooten WM. Advances in the diagnosis and management of neck pain. BMJ 2017;358:j3221.

6. Cohen SP, Raja SN. Pathogenesis, diagnosis, and treatment of lumbar zygapophysial (facet) joint pain. Anesthesiology 2007;106:591-614.

7. Manchikanti L, Pampati V, Fellows B, Bakhit CE. The diagnostic validity and therapeutic value of lumbar facet joint nerve blocks with or without adjuvant agents. Curr Rev Pain 2000;4:337-44.

8. Huang RC, Lim MR, Girardi FP, Cammisa FP Jr. The prevalence of contraindications to total disc replacement in a cohort of lumbar surgical patients. Spine (Phila Pa 1976) 2004;29:2538-41.

9. Weishaupt D, Zanetti M, Boos N, Hodler J. MR imaging and CT in osteoarthritis of the lumbar facet joints. Skeletal Radiol 1999;28:215-9.

10. Manchikanti L, Manchikanti KN, Cash KA, Singh V, Giordano J. Age-related prevalence of facet-joint involvement in chronic neck and low back pain. Pain Physician 2008;11:67-75.

11. Master DL, Eubanks JD, Ahn NU. Prevalence of concurrent lumbar and cervical arthrosis: an anatomic study of cadaveric specimens. Spine (Phila Pa 1976) 2009;34:E272-5.

12. Kim KY, Wang MY. Magnetic resonance image-based morphological predictors of single photon emission computed tomography-positive facet arthropathy in patients with axial back pain. Neurosurgery 2006;59:147-56.

13. Cavanaugh JM, Lu Y, Chen C, Kallakuri S. Pain generation in lumbar and cervical facet joints. J Bone Joint Surg Am 2006;88 Suppl 2:63-7. 